\title{
Three-dimensional culturing models for bone development
}

\section{Opinion}

Three-dimensional (3D) cell culturing system is gaining more interest in cell biology, being more physiological and closer to the natural environment of the cells. For stem cell, 3D culture is of great importance as the proximity of the cells to each other, in spatial conformation, allows for better intercellular communication and enhances differentiation. Monolayer or two-dimensional (2D) cell culture was classically used for studying the differentiation of stem cells into osteoblasts. This technique involves growing of the cells as on solid and impermeable surfaces, such as different polymers of tissue culture plastic. These studies have markedly helped in enhancing our understanding of the molecular characteristics and signaling pathways involved in osteogenesis. ${ }^{1,2}$ Researchers were satisfied by the possible upregulation of the differentiation markers, such as the expression of alkaline phosphatase enzyme and/or the deposition of extracellular calcium. Unfortunately, cells cultured as monolayer lack the complexity as well as the physiological and phenotypical relevance of the tissues. ${ }^{3}$ For osteogenic studies, the deposition of extracellular matrix proteins was always a challenge to validate and similarly the localization of the calcium deposits, either in the matrix or insides apoptotic cells. Osteoblasts are normally embedded in the bone matrix; such environment is not only important for the bone characteristics but also for the full differentiation of osteoblasts into osteocytes. Thus, it is not surprising that the $3 \mathrm{D}$ configuration could be essential for the proper osteogenic differentiation of stem cells. ${ }^{4,5}$ Ferrara et al. studied $2 \mathrm{D}$ versus $3 \mathrm{D}$ culture model of human derived osteoblast, isolated from adult subjects. They found that the production of alkaline phosphatase by $3 \mathrm{D}$ cultures was similar to that of monolayer, while calcification in $3 \mathrm{D}$ cultures was about tenfold that of monolayer. Interestingly, the cells in 3D cultures developed tight junctions and had increased the cell-to-cell contact. ${ }^{6}$ The authors concluded that in-vitro 3D cultures of human osteoblasts can promote further differentiation up to mineral deposition. These results highlighted the crucial role for the interaction between osteoblasts and the surrounding environment. Similar conclusions were reached by kale et al, as the authors reported the spontaneous formation of bone nodules upon maturation of the cells in monolayer under the effect of transforming growth factor beta. ${ }^{7}$

The use of scaffolds was one of the earliest models for 3D culture. Scaffolds can be natural, such as decellularized primary tissues, or synthetic. The first type is based on the decellularization of donated primary tissue, followed by seeding of the patient's cells. The new composite will keep the shape and the matrix pattern of the donor, while presents the antigens of the patient. Bones can be donated by a deceased person or can be even from animal origin. The presence of such framework, with the help of the media additives, enhances the differentiation of the stem cells. This approach is of current clinical use in dentistry, especially to restore the bone mass around dental implants. ${ }^{8}$ On the other hand, synthetic scaffold can be synthesized from various materials according to the differentiation target. Synthetic scaffolds can be shaped according to the downstream application, up to filling a bone defect in a patient. Scaffolds can provide protection of
Volume 4 Issue 4 - 2018

\author{
Ahmed A Nugud,' Ibrahim El-Serafi, ${ }^{2}$ Ahmed \\ T El-Serafi ${ }^{3}$ \\ 'General Pediatric Department, Al-Jalila Hospital, UAE \\ ${ }^{2}$ Oncology-Pathology Department, Karoliniska Institutet, \\ Sweden \\ ${ }^{3}$ Basic Medical Sciences Department, College of Medicine, \\ University of Sharjah, UAE
}

Correspondence: Ahmed El-Serafi, Basic Medical Sciences Dep, College of Medicine, University of Sharjah, Sharjah University City, PO Box 27272, Sharjah, UAE,

Email ahmed.elsarafil@med.suez.edu.eg

Received: December II, 2018 | Published: December 17, 2018

the cells, support cellular growth and enhance differentiation. Various materials can be used to prepare scaffolds for bone regeneration, including ceramic, calcium phosphate, calcium hydroxyapatiet or a combination of them. ${ }^{9}$ One of the major limitations of scaffolds is the long-term stability. In addition to the loss of the scaffold role, the degradation products could be toxic and associated with changes of the cell characteristics and phenotype. ${ }^{10,11}$

Scaffold-free models of 3D culture were classically involving few thousands of cells that led to microscopic aggregates. Alternatively, pellet culture system can use up to one million cell and produce a spheroid of about $1 \mathrm{~mm}$ in diameter. The latter would have the same advantages of the $3 \mathrm{D}$ culture and allows the cells to secrete the extracellular proteins that will work as natural scaffolds. Fetal skeletal cell provided another level of evidence regarding the physiological nature of the pellet culture system. When these cells were cultured as pellets, the cells reorganized themselves in three distinct zones; outer fibrous layer, middle osteogenic shell and central chondrogenic core. Interestingly, this arrangement was similar to the cross section of the fetal bone. Thus, such 3D environment provided the milieu for the physiological differentiation and arrangement of the cells, including the presence of three types of cells while cultured in osteogenic media. ${ }^{12}$ This 3D ex-vivo model can be used to test different factors that trigger bone and cartilage development as well as various therapeutic compounds that can have their primary or side effects on bones. Nevertheless, the applications of this model can be extended to examine the effects of mechanical forces, growth factors, vitamins, toxins or pollutants on bone development. In conclusion, various forms of $3 \mathrm{D}$ culturing system can be applied to achieve physiological osteogenic differentiation as well as for drug testing studies. There are many advantages of 3D culture, including the possibility of matrix characterization using histological stains and immunocytochemistry. This model of culture provides a physiological solution that can help in the reduction of the animals use in research. With further improvement in the scaffold biocompatibility, constructs could be formulated ex-vivo and transferred to the patients as a regenerative 
solution for lost bone segments. Combination of several pellets can fill in a bone gap or help in the secondary fixation of bone prothesis. With the advances of induced pluripotent stem cell, the scaffold-free pellet system can be in the frontline. Large number of cells will be easily prepared for the patient, which will allow for the production of many pellets that can fit a large gap size. The presence of spaces between the pellets will give access to neovascularization, which will ensure their integrity. Thus, the 3D culture system will not help only in studying the biological basis of stem cell differentiation into osteoblasts or the effect of various chemical or physical challenges on bone development, but will also help in the future clinical application of bone regeneration.

\section{Acknowledgments}

None.

\section{Conflicts of interest}

Author declares that there is no conflicts of interest.

\section{References}

1. Friedl P. Prespecification and plasticity: shifting mechanisms of cell migration. Curr Opin Cell Biol. 2004;16(1):14-23.

2. Walpita D, Hay E. Studying actin-dependent processes in tissue culture. Nat Rev Mol Cell Biol. 2002;3(2):137-141.

3. Pampaloni F, Reynaud E, Stelzer E. The third dimension bridges the gap between cell culture and live tissue. Nat Rev Mol Cell Biol. 2007;8(10):839-845.
4. Thompson W, Uzer G, Brobst K, et al. Osteocyte specific responses to soluble and mechanical stimuli in a stem cell derived culture model. Sci Rep. 2015;5:11049.

5. Xiong J, Onal M, Jilka R, et al. Matrix-embedded cells control osteoclast formation. Nat Med. 2011;17(10):1235-1241.

6. Ferrera D, Poggi S, Biassoni C, et al. Three-dimensional cultures of normal human osteoblasts: proliferation and differentiation potential in vitro and upon ectopic implantation in nude mice. Bone. 2002;30(5):718 725 .

7. Kale S, Biermann S, Edwards C, et al. Three-dimensional cellular development is essential for ex vivo formation of human bone. Nat Biotech. 2000;18(9):954-958.

8. Malinin TI, Temple HT, Garg AK. Bone Allografts in Dentistry: A Review. Dentistry. 2014;4:1000199.

9. Luongo F, Mangano FG, Macchi A, et al. Custom-Made Synthetic Scaffolds for Bone Reconstruction: A Retrospective, Multicenter Clinical Study on 15 Patients. Biomed Res Int. 2016;2016:5862586.

10. El-Serafi A, El-Serafi I, Elmasry M, et al. Skin regeneration in three dimensions, current status, challenges and opportunities. Differentiation. 2017;96:26-29.

11. Ferrera D, Poggi S, Biassoni C, et al. Three-dimensional cultures of normal human osteoblasts: proliferation and differentiation potential in vitro and upon ectopic implantation in nude mice. Bone. 2002;30(5):718725 .

12. El-Serafi A, Wilson D, Roach H, et al. Developmental plasticity of human foetal femur-derived cells in pellet culture: self assembly of an osteoid shell around a cartilaginous core. Eur Cell Mater. 2011;21:558-567. 